\title{
Intersexualidad en el camarón excavador Parastacus pugnax (Poeppig, 1835) (Decapoda, Parastacidae)
}

\author{
Erich H. Rudolph \\ Departamento de Ciencias Básicas, Universidad de Los Lagos \\ Casilla 933, Osorno, Chile
}

\begin{abstract}
RESUMEN. Se estudió el problema de intersexualidad en el camarón excavador Parastacus pugnax (Poeppig, 1835), a través del examen de los caracteres sexuales de 538 ejemplares capturados en Nehuentúe, Chile. Se demostró que en esta especie machos y hembras son intersexo, con gonoporos supernumerarios y con una gónada única -masculina o femenina según el caso- de la cual emergen gonoductos de ambos sexos hacia los respectivos gonoporos. En los machos los oviductos son rudimentarios y los gonoporos femeninos son semielipsoidales, con una cubierta muy calcificada. En las hembras los espermiductos son rudimentarios y los gonoporos femeninos son elipsoidales y cubiertos por una membrana no calcificada o sólo parcialmente calcificada. En las hembras se identificaron caracteres sexuales secundarios permanentes asociados a la incubación de los huevos. Se discute el valor adaptativo de este tipo de genitalia en el contexto de la historia evolutiva de los Parastacidae.
\end{abstract}

Palabras claves: gonoporos supernumerarios, Parastacus pugnax, intersexualidad, Parastacidae, Chile.

\section{Intersexuality in the burrowing crayfish Parastacus pugnax (Poeppig, 1835) (Decapoda, Parastacidae)}

\begin{abstract}
The problem of intersexuality was studied in the burrowing crayfish Parastacus pugnax (Poeppig, 1835) by examination of sexual characters of 538 specimens captured in Nehuentúe, Chile. It was demostrated that in this species both male and female are intersex, with supernumerary gonopores and with single gonad -either male or female- from which emerge gonoducts of both sexes to the respective gonopores. In males the oviducts are rudimentary and female gonopores are semiellipsoidal, with a very calcified cover. In females the sperm ducts are rudimentary and female gonopores are ellipsoidal and covered by a noncalcified membrane or just partially calcified. Permanent secondary sexual characters associated with incubation of eggs were identified in females. The adaptive value of this type of genitalia is discussed in the evolutionary historical context of Parastacidae.
\end{abstract}

Key words: supernumerary gonopores, Parastacus pugnax, intersexuality, Parastacidae, Chile.

\section{INTRODUCCION}

La presencia de gonoporos supernumerarios en Parastacus se conoce desde que Von Martens (1869) describió dos especies de camarones brasileros: Astacus pilimanus (=Parastacus pilimanus) y A. brasiliensis (=P. brasiliensis), haciendo notar la presencia de gonoporos femeninos en los machos de ambas especies. Faxon (1898) estableció que no sólo los representantes de estas dos especies, sino también los de $P$. saffordi Faxon, $P$. varicosus Faxon, P. defossus Faxon y P. hassleri Faxon (= P. pugnax Poeppig), tienen doble juego de orificios genitales. Esto sirvió de base para que autores posteriores calificaran a Parastacus como el único género de Parastacidae con gonoporos de hembra y macho en un mismo individuo (Riek, 1971; Hobbs, 1974 y 1991). Sin embargo Rudolph (1990) señaló que $P$. nicoleti (Philippi) es una excepción, ya que sólo algunos ejemplares de esta especie tienen gonoporos de ambos sexos. Los estudios destinados a conocer el significado funcional de los gonoporos supernumerarios han sido escasos, involucrando un número reducido de ejemplares de: $P$. brasiliensis, P. hassleri, $P$. saffordi, $P$. defossus, $P$. varicosus y $P$. pilimanus (Von Ihering, 1893; Lönnberg, 1898; 
Hay, 1905; Turner, 1935; Thompson, 1982). De estos autores, Lönnberg (1898) y Hay (1905), postularon un probable hermafroditismo en Parastacus; los restantes lo descartaron. Rudolph (1995b) analizó los caracteres sexuales externos e internos de 473 especímenes de $P$. nicoleti, concluyendo que en esta especie hay dos tipos sexuales básicos, hembras primarias y hermafroditas protándricos.

$P$. pugnax es un camarón excavador que habita terrenos semipantanosos, desde el río Aconcagua (32 50 'S; $70^{\circ} 59^{\prime} \mathrm{W}$ ) hasta la localidad de Carahue (38 $\left.40^{\prime} \mathrm{S} ; 7^{\circ} 09^{\prime} \mathrm{W}\right)$, en la zona centro-sur de Chile. Las poblaciones de esta especie, en particular aquellas que viven en la zona central de Chile, soportan una intensa explotación para consumo humano (Rudolph et al., 1991). El conocimiento biológico de $P$. pugnax es limitado y se circunscribe a aspectos de índole taxonómico, de distribución y de descripción de las primeras etapas de su desarrollo ontogenético en condiciones de laboratorio (Philippi, 1882; Faxon, 1898; Holthuis, 1952; Bahamonde y López, 1963; Riek, 1971; Manning y Hobbs, 1977; Rudolph y Ríos, 1987; Hobbs, 1989 y 1991; Arias y Muñoz, 1991). La existencia de gonoporos supernumerarios en $P$. pugnax, fue analizada a través de disecciones sólo por Lönnberg (1898), y por Turner (1935). Las conclusiones de estos autores fueron contrapuestas. Mientras Lönnberg postuló un probable hermafroditismo, Turner rechazó esta posibilidad. Desde entonces no se ha realizado ningún estudio, destinado a determinar si $P$. pugnax es una especie hermafrodita o gonocórica con individuos intersexo. Esto ha impedido avanzar en el conocimiento de la biología reproductiva y poblacional de esta especie. En este artículo se describen los caracteres sexuales externos e internos de $P$. pugnax, con la finalidad de conocer el tipo de sexualidad de este parastácido chileno.

\section{MATERIALES Y METODOS}

Especímenes de $P$. pugnax fueron recolectados en la localidad de Nehuentúe (38²4'S; 73²3'W), Provincia de Cautín, entre septiembre de 1994 y octubre de 1995. Se realizaron 7 muestreos, recolectándose un total de 538 individuos. Los ejemplares se capturaron con una bomba de vacío parcial, separándolos en bolsas de polietileno según la galería que habitaban al momento de ser capturados y siendo fijados en Bouin acuoso por 48 horas. En el laboratorio, se examinaron los gonoporos de cada ejemplar con un microscopio estereoscópico provisto de cámara de dibujo. Esto permitió clasificarlos en dos formas sexuales. En cada individuo se midieron - con un vernier de 0,01 $\mathrm{cm}$ de precisión o un micrómetro ocular adaptado a la lupa- las siguientes dimensiones: longitud estándar del cefalotórax (LEC), longitud de los somitos abdominales, ancho de los somitos abdominales 2 al 6, ancho y largo del telson, ancho y largo del endopodito del urópodo, ancho y largo del exopodito del urópodo, alto de las pleuras de los somitos abdominales 3 al 5, longitud de la pleura del somito abdominal 2, y alto de la papila genital masculina. Para esto último se extrajeron los podómeros basales de los apéndices del XIV segmento corporal; luego de ubicarlos en vista caudal se midió en línea recta desde el ápice de la papila hasta la unión de la coxa con su respectiva membrana articular. Los valores de estas mediciones se estandarizaron, dividiéndolos por el correspondiente valor de la longitud cefalotorácica. Se seleccionaron individuos de cada forma sexual, de tallas similares, a fin de comparar los promedios de las variables morfométricas mediante la prueba "t" de Student.

La proporción sexual se evaluó aplicando el test de chi-cuadrado con la corrección de Yates. En las hembras ovígeras se contaron los huevos, embriones y/o juveniles. Para identificar las etapas del desarrollo ontogenético se emplearon las descripciones de Rudolph y Ríos (1987). Se seleccionaron algunos ejemplares de cada forma sexual, para describir sus caracteres sexuales externos y luego disecarlos para analizar la anatomía de la gónada y gonoductos. Estas estructuras fueron removidas para su estudio histológico, el que se inició con la deshidratación en una serie de alcoholes e inclusión en parafina. Se efectuaron cortes de 7 $\mathrm{mm}$ de espesor, los cuales se tiñeron con hematoxilina-eosina. Para reconocer y caracterizar la histología de la gónada, se emplearon las descripciones y fotomicrografías de Payen (1973), de Amato y Payen (1976 y 1978) en Astacus leptodactylus Eschscholtz, y de Rudolph (1995b) en $P$. nicoleti. También se realizaron algunas observaciones microscópicas de macerados testiculares en fresco.

\section{RESULTADOS}

\section{Descripción de los caracteres sexuales externos}

El examen de los caracteres sexuales externos, de todos los ejemplares capturados, permitió distinguir 
dos formas sexuales:

Intersexos 1: ejemplares que presentan esbozos de gonoporos femeninos de forma semielipsoidal, muy calcificados, sin pilosidad ni rastro de abertura, en las coxas del tercer par de pereiópodos. Además, presentan una papila fija, calcificada, en cuyo extremo apical se abre el gonoporo masculino, en el borde interno de las coxas del quinto par de pereiópodos (Fig. 1A). La talla de estos intersexos fluctuó entre 6,6 y 47,4 mm de longitud cefalotorácica.

Intersexos 2: ejemplares con gonoporos femeninos de forma elipsoidal, rodeados por abundante y larga pilosidad y cubiertos por una membrana que puede ser fina, semitransparente y algo convexa, o bien presentarse parcialmente calcificada, plana y no transparente. Presentan además, gonoporos masculinos similares en su morfología a los descritos en los intersexos 1 (Fig. 1B). La longitud cefalotorácica de estos ejemplares fluctuó entre 26,2 y 45,4 mm.

\section{Morfología de gónadas y gonoductos}

La gónada de $P$. pugnax, está formada por dos túbulos paralelos. En ellos se distingue una región anterior pequeña, adosada al hepatopácreas anterior en sentido dorsoventral y otra posterior situada por debajo del corazón, que se extiende en dirección caudal por encima del hepatopáncreas posterior. De esta última región emergen los gonoductos. Los oviductos son planos y del mismo ancho en toda su extensión. Se originan en la zona angular que forman las porciones gonádicas anterior y posterior. Desde allí se dirigen rectamente hacia las coxas del tercer par de pereiópodos. Los espermiductos se originan inmediatamente por detrás del punto de origen de los oviductos, pero sólo se separan de los túbulos gonádicos cuando han alcanzado la porción posterior de ellos. A partir de allí descienden hacia las coxas del quinto par de pereiópodos. El tercio proximal de los vasos deferentes es sinuoso a diferencia de los dos tercios restantes que carecen de estas sinuosidades. Todos los especímenes de $P$. pugnax analizados en este estudio, presentan gonoductos de ambos sexos conectados a una gónada completamente femenina o masculina según el caso. Cuando la gónada es un testículo (Fig. 2A), los oviductos son delgados $(\mathrm{n}=12 ; \mathrm{x}=0,41 \mathrm{~mm}$; $\mathrm{DS}=$ $\pm 0,13$ ) y los espermiductos son de textura firme, cilíndricos, sus porciones sinuosas son delgadas y de mayor diámetro las restantes $(n=12 ; x=0,89 \mathrm{~mm}$; $\mathrm{DS}= \pm 0,20)$. En la región subterminal de ellos se

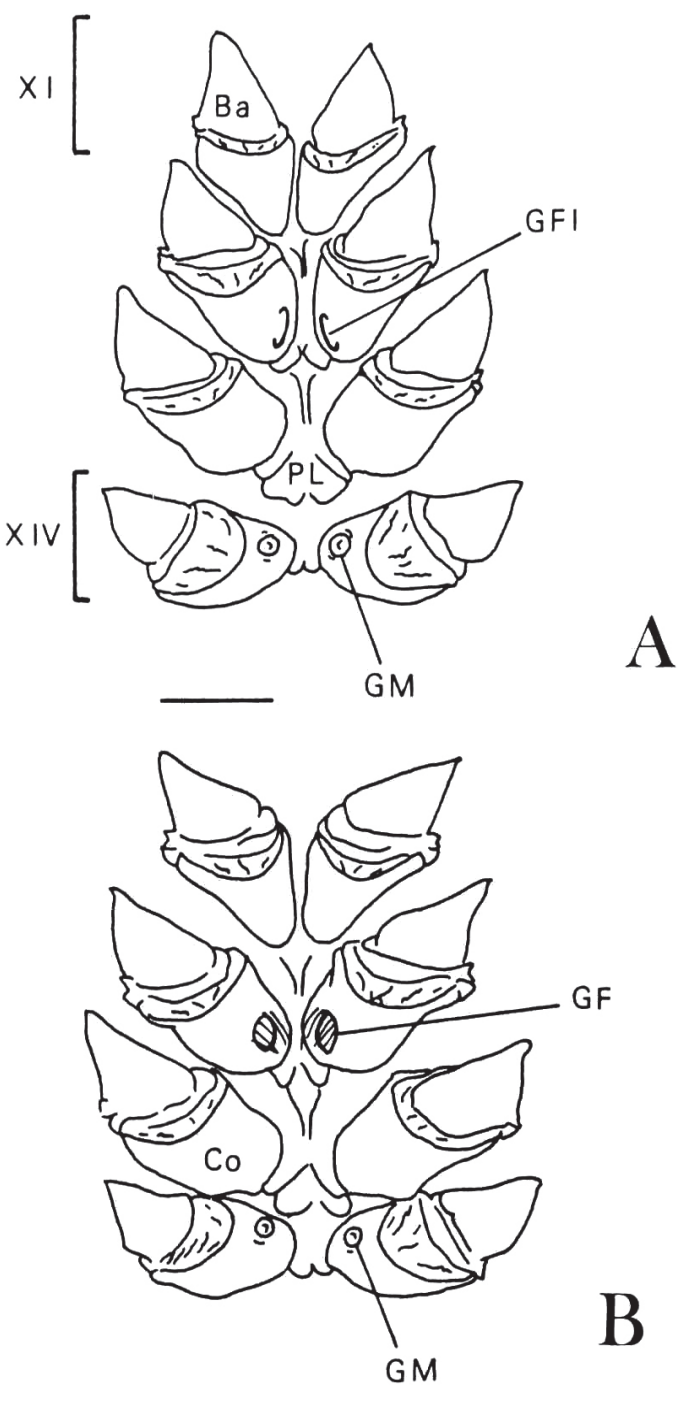

Figura 1. P pugnax. Región esternal y coxas de los pereiópodos asociados a los segmentos corporales XI a XIV. A: intersexo 1; B: intersexo 2. Escala $=0,5 \mathrm{~cm}$. (Ba:basipodito; Co:coxopodito; GFI: gonoporo femenino; GM: gonoporo masculino; PL: proceso lateral; XI: segmento corporal XI; XIV: segmento corporal XIV).

Figure 1. P. pugnax. Sternal region and coxa of pereiopods associated with body segments XI through XIV.A: intersex 1; B intersex 2. Scale= $0,5 \mathrm{~cm}$. (Ba: basipodite; Co: coxopodite; GFI: incomplete female gonopore;GF: female gonopore; GM: male gonopore;PL: lateral process; XI: body segment XI; XIV: body segment XIV). 

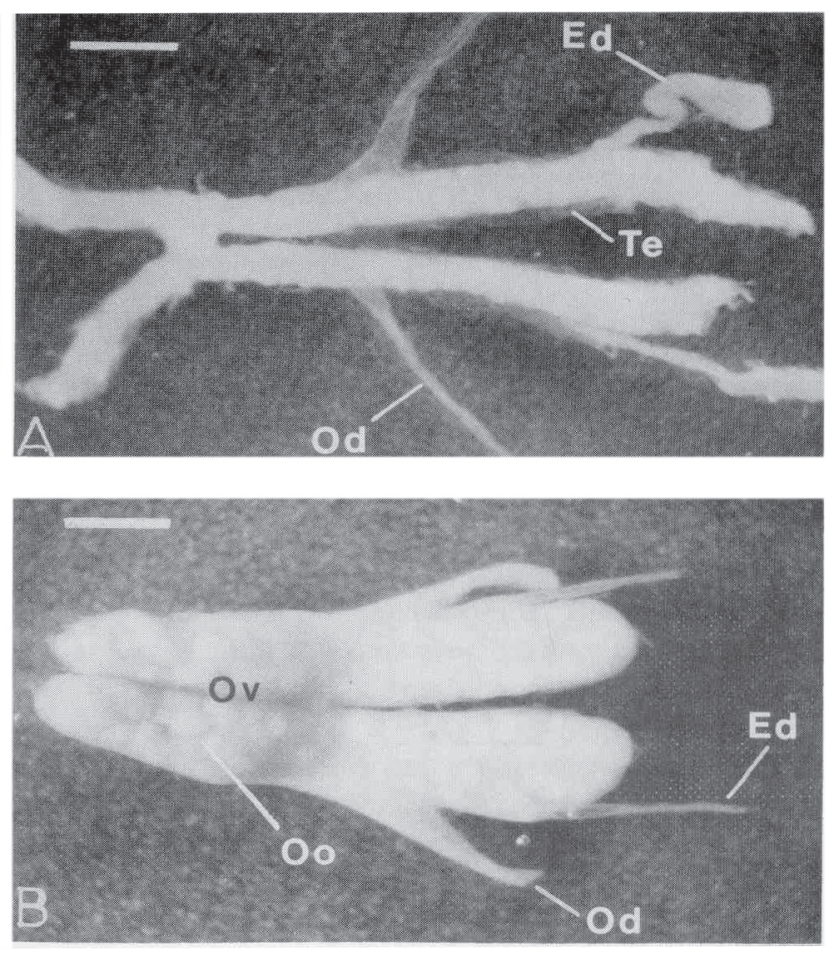

Figura 2. P. pugnax. Características anatómicas de la genitalia. A: genitalia masculina; B: genitalia femenina. Escala $=2 \mathrm{~mm}$. (Te: testículo; Ed: espermiducto; Ov: ovario; Od: oviducto; Oo: ovocitos).

Figure 2. P. pugnax. Genital anatomical features. A: male genitalia; $B$ : female genitalia $S c a l e=2 \mathrm{~mm}$. $($ Te : testes; Ed: spermiduct; Ov : ovary; Od : oviduct; Oo : ovocites).

ubica la glándula androgénica y en sus extremos terminales existe una zona eyaculatriz, musculosa y más ancha que las porciones precedentes. En cambio, cuando la gónada es un ovario (Fig. 2B), los oviductos son anchos ( $\mathrm{n}=16$; $\mathrm{x}=1,13 \mathrm{~mm} ; \mathrm{DS}= \pm 0,441)$ y los vasos deferentes planos $\mathrm{y}$ finos $(n=16 ; x=0,20 \mathrm{~mm} ; \mathrm{DS}= \pm 0,07)$. En la región subterminal de este tipo de espermiductos no fue posible observar la glándula androgénica. En los ovarios se observa: pared ovárica, gonias, ovocitos y células foliculares. Su color fluctúa entre blanco y anaranjado, según la etapa de vitelogénesis en que se encuentren sus ovocitos (Fig. 3A). Los testículos son blancos, y en ellos se observan: canales colectores y acinos testiculares, en estos últimos se distinguen una vaina conjuntiva externa, gonias, espermatocitos en división meiótica, espermátidas y espermatozoides (Fig. 3B).

Bajo los $26 \mathrm{~mm}$ de longitud cefalotorácica, todos los individuos analizados $(\mathrm{n}=326)$ presentan gonoporos supernumerarios típicos de los intersexo 1. Sin embargo la disección de estos ejemplares demostró que sus gónadas ya estaban diferenciadas, encontrándose que el $60,4 \%$ de 1 os ejemplares eran machos intersexo y el $39,6 \%$ restante hembras intersexo. Sobre los $26 \mathrm{~mm}$ de LEC se encontraron 212 ejemplares, de ellos $125(59 \%)$ presentaban gonoporos supernumerarios característicos de los intersexo 1. Los 87 ejemplares restantes (41\%), tenían gonoporos supernumerarios propios de los intersexo 2 . La disección de ellos demostró que el 53,8\% (n=114) eran machos y el 46,2\% (n=98) hembras. Esto indica que sobre los $26 \mathrm{~mm}$ de LEC, el 5,2\% de los intersexo 1 son hembras.

\section{Caracteres sexuales secundarios}

No se encontraron diferencias significativas entre los abdómenes de los machos y hembras, de tallas inferiores a $26 \mathrm{~mm}$ de LEC (Tabla 1). La única diferencia significativa $(p \leq 0,01)$ en estos individuos, se obtuvo al comparar las medias del alto de las papilas genitales masculinas. En los machos la papila genital masculina es más alta, que la respectiva papila existente en las hembras (Tabla 2).

Al comparar los abdómenes de los machos y hembras de longitud cefalotorácica superior a $26 \mathrm{~mm}$, se encontraron diferencias significativas $(\mathrm{p} \leq 0,01)$ en 13 de las 16 medias analizadas. El abdomen de las hembras con respecto al abdomen de los machos, es significativamente más ancho, sus pleuras significativamente más altas y las ramas internas y externas de los urópodos son significativamente más largas y más anchas. Sólo la longitud de los somitos abdominales y el largo y ancho del telson son similares en ambos sexos (Tabla 3). Los machos de longitud cefalotorácica superior a $26 \mathrm{~mm}$, presentan una papila genital masculina significativamente $(\mathrm{p} \leq 0,01)$ más alta, que la papila fálica de las hembras (Tabla 2).

\section{Distribución de frecuencia por clase de talla}

No se capturaron ejemplares bajo los $6,6 \mathrm{~mm}$ ni sobre los 47,4 mm de LEC. Tampoco se encontraron individuos entre 20 y $25 \mathrm{~mm}$ de longitud cefalotorácica en noviembre, junio y octubre. Además, el porcentaje de 
Tabla 1. Medias y desviaciones estándar de los caracteres morfológicos de machos y hembras de P.pugnax de tallas inferiores a $26 \mathrm{~mm}$. de longitud estándar del cefalotórax (LEC), con el nivel de significancia del test $\mathrm{T} . \mathrm{L}=$ Longitud, $\mathrm{A}=$ ancho, $\mathrm{Al}=$ alto.

Table 1. Mean and standard deviations of morphological characters of males and females of $P$. pugnax of less than $26 \mathrm{~mm}$ cephalothoraxic standard length (LEC), with the corresponding significance level T test. $\mathrm{L}=$ length, $\mathrm{A}=$ width, $\mathrm{A1}=$ height.

\begin{tabular}{|c|c|c|c|c|c|}
\hline \multirow[t]{2}{*}{ Carácter morfológico } & \multicolumn{2}{|c|}{$\begin{array}{c}\text { Machos } \\
n=17 \\
\bar{x} \text { LEC }=12,9\end{array}$} & \multicolumn{2}{|c|}{$\begin{array}{cc} & \text { Hembras } \\
n=18 \\
\bar{x} \quad & \operatorname{LEC}=12,5 \\
\end{array}$} & \multirow{2}{*}{$\begin{array}{c}\text { Significancia } \\
\text { n.s. }\end{array}$} \\
\hline & $\overline{\mathbf{x}}$ & \pm D.S. & $\overline{\mathbf{x}}$ & \pm D.S. & \\
\hline L. somitos abdominales & 0,735 & 0,0447 & 0,743 & 0,0619 & n.s. \\
\hline A. somito 2 & 0,407 & 0,0201 & 0,418 & 0,0154 & n.s. \\
\hline A. somito 3 & 0,410 & 0,025 & 0,422 & 0,0182 & n.s. \\
\hline A. somito 4 & 0,403 & 0,0293 & 0,415 & 0,0217 & n.s. \\
\hline A. somito 5 & 0,392 & 0,0245 & 0,399 & 0,0292 & n.s. \\
\hline A. somito 6 & 0,365 & 0,0194 & 0,370 & 0,0230 & n.s. \\
\hline A. telson & 0,258 & 0,0091 & 0,259 & 0,0129 & n.s. \\
\hline L. telson & 0,314 & 0,0181 & 0,309 & 0,0171 & n.s. \\
\hline L. endopodito urópodo & 0,264 & 0,0200 & 0,268 & 0,0157 & n.s. \\
\hline L. exopodito urópodo & 0,307 & 0,0217 & 0,316 & 0,0133 & n.s. \\
\hline A. endopodito urópodo & 0,200 & 0,0123 & 0,199 & 0,0091 & n.s. \\
\hline A. exopodito urópodo & 0,214 & 0,0115 & 0,219 & 0,0088 & n.s. \\
\hline Al. pleura somito 3 & 0,056 & 0,0039 & 0,056 & 0,0056 & n.s. \\
\hline Al. pleura somito 4 & 0,061 & 0,0051 & 0,060 & 0,0055 & n.s. \\
\hline Al. pleura somito 5 & 0,061 & 0,0050 & 0,062 & 0,0076 & n.s. \\
\hline L. pleura somito 2 & 0,207 & 0,0088 & 0,210 & 0,0091 & n.s. \\
\hline
\end{tabular}

Tabla 2. Medias y desviaciones estándar del alto del la papila genital masculina en machos y hembras de $P$. pugnax, de tallas inferiores y superiores a $26 \mathrm{~mm}$ de longitud estándar del cefalotórax (LEC), con el nivel de significancia del test $\mathbf{T}$. APGM = alto papila masculina.

Table 2. Mean and standard deviations of $P$. pugnax males and females genital papillae height, for lenght less than and over $26 \mathrm{~mm}$ cephalothoraxic standard length (LEC), with its corresponding significance level T test, APGM = height of male papillae.

\begin{tabular}{|c|c|c|c|c|c|c|c|}
\hline \multirow[t]{2}{*}{ APGM } & \multicolumn{4}{|c|}{ Machos } & \multicolumn{2}{|r|}{ Hembras } & \multirow[t]{2}{*}{ Significancia } \\
\hline & $\mathrm{n}=32$ & $\bar{x}$ & $\mathrm{LEC}=12,9$ & $\mathrm{n}=25$ & $\bar{x}$ & $\mathrm{LEC}=12,1$ & \\
\hline \multirow[t]{2}{*}{$\overline{\mathrm{x}}$} & & \multicolumn{3}{|c|}{0,0621} & \multicolumn{2}{|r|}{0,0525} & \\
\hline & & \multirow{2}{*}{\multicolumn{2}{|c|}{0,0047}} & & & & $\mathrm{p}<0,01$ \\
\hline \multirow[t]{2}{*}{ \pm D.S. } & & & & & \multicolumn{2}{|r|}{0,0035} & \\
\hline & $\mathrm{n}=14$ & $\bar{x}$ & $\mathrm{LEC}=40,8$ & $\mathrm{n}=16$ & $\bar{x}$ & $\mathrm{LEC}=38,7$ & \\
\hline \multirow[t]{2}{*}{$\overline{\mathrm{x}}$} & & \multirow{2}{*}{\multicolumn{2}{|c|}{0,084}} & & \multicolumn{2}{|r|}{0,0774} & \\
\hline & & & & & & & $\mathrm{p}<0,01$ \\
\hline \pm D.S. & & \multicolumn{2}{|r|}{0,00606} & & \multicolumn{2}{|r|}{0,00553} & \\
\hline
\end{tabular}



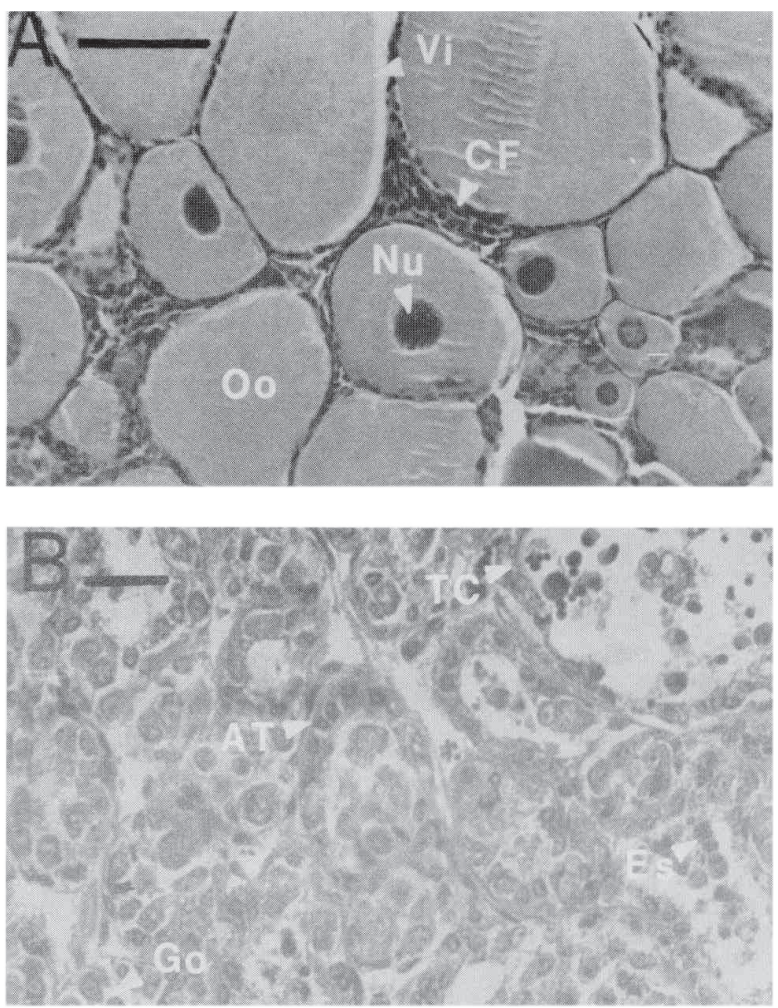

Figura 3. P. pugnax. Características histológicas de las gónadas. A: tejido ovárico; $B$ : tejido testicular. Escala = A, $130 \mu \mathrm{m} ; \mathrm{B}, 34 \mu \mathrm{m}$. (CF: células foliculares; Oo: ovocito; Vi: vitelo; Nu: núcleo; TC: túbulo colector; AT: acino testicular; Es: espermatozoos; Go: gonias.

Figure 3. P. pugnax. Gonadal histological features. A: ovaric tissue; B: testicular tissue. Scale $=\mathbf{A}: 130$ $\mu \mathrm{m} ; \mathrm{B}: 34 \mu \mathrm{m}$. (CF: folicular cells; Oo: ovocite; Vi: vitellum; nucleus; TC: collector tubule, AT: testicular acine; Es; spermatozoids; Go: gonies).

individuos en este rango fue inferior al $2,5 \%$, en septiembre $(2,4 \%)$, abril $(2,2 \%)$ y agosto $(1,1 \%)$. En enero se encontró la mayor cantidad de ejemplares en este rango $(11,5 \%)$. No se encontraron hembras entre los 20 y $25 \mathrm{~mm}$ de LEC, en ninguno de los muestreos realizados. Los mayores porcentajes de especímenes bajo los $25 \mathrm{~mm}$ de LEC (juveniles o prepúberes), se observan en septiembre, abril, junio y agosto, y los menores en noviembre, enero y octubre. Estos últimos 3 meses, fueron los únicos en los cuales se capturaron hembras ovígeras. En los muestreos efectuados en abril y junio, se capturó la menor cantidad de ejemplares de longitud cefalotorácica superior a $25 \mathrm{~mm}$ (Fig. 4).

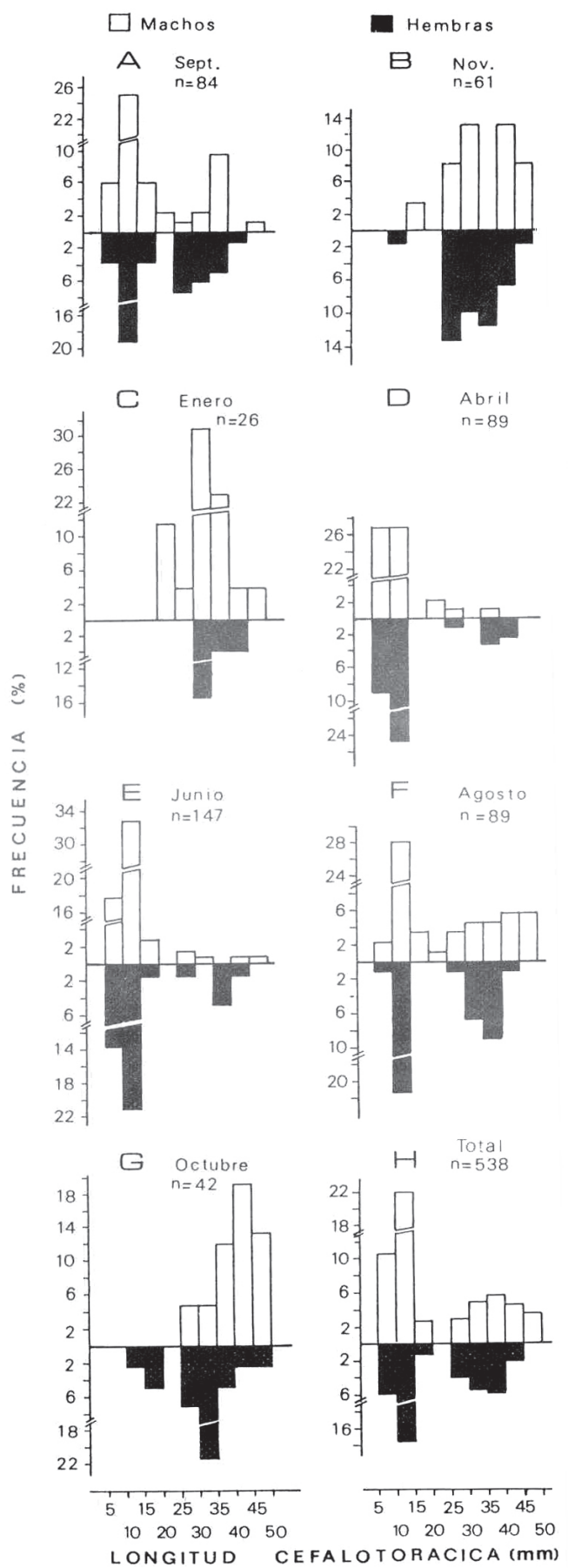

Figura 4. $P$. pugnax. Distribución de frecuencia de machos y hembras por clase de talla y por muestreo.

Figure 4. $P$. pugnax. Male and female frequency distribution by length class and sample. 


\section{Tamaño y forma sexual de las hembras ovígeras}

Todas las hembras ovígeras colectadas midieron más de $26 \mathrm{~mm}$ de longitud cefalotorácica. La más pequeña midió $30,6 \mathrm{~mm}$ y la más grande $45,1 \mathrm{~mm}$. Además las 23 hembras ovígeras capturadas, presentaban gonoporos supernumerarios típicos de los intersexo 2 (Tabla 4). Al disecar 15 de estas hembras, se observó en sus ovarios la presencia de ovocitos en distintas etapas de vitelogénesis, los cuales en promedio, eran de menor tamaño que los ovocitos de las hembras no ovígeras (Tabla 5). Esto permite suponer que las hembras de esta especie probablemente tengan desoves múltiples.

\section{Proporción sexual}

De los 538 ejemplares colectados, 311 fueron machos intersexo y 227 hembras intersexo, lo que equivale a una proporción sexual 1,4:1. Este valor difiere significativamente de una proporción sexual del tipo $1: 1(\bar{x}=11,51 ; \mathrm{p}<0,05)$; lo mismo ocurrió con la proporción de sexos registrada en el tercer muestreo (enero) $(\overline{\mathrm{x}}=7,23 ; \mathrm{p}<0,05)$.

La proporción sexual en especímenes de talla inferior a los $25 \mathrm{~mm}$ de longitud cefalotorácica fue 197 machos y 129 hembras (1,5:1), la cual también difiere significativamente de una proporción $1: 1$ $(\bar{x}=12,78 ; p<0,05)$. Sin embargo, en especímenes de talla superior a $25 \mathrm{~mm}$ de LEC la proporción fue 114 machos y 98 hembras $(1,2: 1)$, la cual no difirió significativamente de una proporción 1:1.

$\mathrm{Si}$ se analiza la proporción de sexos en cada clase de talla, se observa que en todas aquellas menores a $26 \mathrm{~mm}$ de LEC es mayor el número de machos. Lo mismo ocurre en las clases de talla más grandes, esto es: 40,0-44,9 y 45,0-49,9 mm. Sin embargo, en las clases de tallas comprendidas entre los 25 y $40 \mathrm{~mm}$ se aprecia un mayor número de hembras. No se encontraron diferencias significativas con respecto a una proporción 1:1 en ninguna de las clases de talla, excepto entre 20,0-24,9 y 45,0-49,9 $\mathrm{mm}$ de longitud cefalotorácica.

Tabla 3. Medias y desviaciones estándar de los caracteres morfológicos de machos y hembras de $\boldsymbol{P}$. pugnax de tallas superiores a $26 \mathrm{~mm}$ de longitud estándar del cefalotórax (LEC), con el nivel de significancia del test $\mathrm{T} . \mathrm{L}=$ longitud, $\mathrm{A}=$ ancho, $\mathrm{A1}=$ alto.

Table 3. Mean and standard deviations of morphological characters of males and females of $P$. pugnax greater then $26 \mathrm{~mm}$ cephalothoraxic standard lenght (LEC), with its corresponding significance level $\mathrm{T}$ test. $\mathrm{L}=$ length, $\mathrm{A}=$ width, $\mathrm{A} 1=$ height.

\begin{tabular}{|c|c|c|c|c|c|}
\hline \multirow[t]{2}{*}{ Carácter morfológico } & \multicolumn{2}{|c|}{$\begin{array}{c}\text { Machos } \\
n=17 \\
\overline{\mathbf{x}} \quad \mathbf{L E C}=\mathbf{3 2 , 9}\end{array}$} & \multicolumn{2}{|c|}{$\begin{array}{c}\text { Hembras } \\
n=18 \\
\bar{x} \quad \text { LEC }=34,4\end{array}$} & \multirow{2}{*}{$\begin{array}{r}\text { Significancia } \\
\text { n.s. }\end{array}$} \\
\hline & $\overline{\mathbf{x}}$ & D.S. & $\overline{\mathbf{x}}$ & D.S. & \\
\hline L. somitos abdominales & 0,769 & 0,0473 & 0,763 & 0,0380 & n.s. \\
\hline A. somito 2 & 0,409 & 0,0114 & 0,427 & 0,0194 & $\mathrm{p}<0,01$ \\
\hline A. somito 3 & 0,423 & 0,0129 & 0,442 & 0,0236 & $\mathrm{p}<0,01$ \\
\hline A. somito 4 & 0,421 & 0,0140 & 0,442 & 0,0233 & $\mathrm{p}<0,01$ \\
\hline A. somito 5 & 0,403 & 0,0105 & 0,421 & 0,0207 & $\mathrm{p}<0.01$ \\
\hline A. somito 6 & 0,368 & 0,0099 & 0,389 & 0,0160 & $\mathrm{p}<0,01$ \\
\hline A. telson & 0,249 & 0,0129 & 0,254 & 0,0090 & n.s. \\
\hline L. telson & 0,303 & 0,0209 & 0,313 & 0,0122 & n.s. \\
\hline L. endopodito urópodo & 0,247 & 0,0086 & 0,280 & 0,0153 & $\mathrm{p}<0,01$ \\
\hline L. exopodito urópodo & 0,285 & 0,0108 & 0,308 & 0,0172 & $\mathrm{p}<0,01$ \\
\hline A. endopodito urópodo & 0,212 & 0,0075 & 0,232 & 0,0143 & $\mathrm{p}<0,01$ \\
\hline A. exopodito urópodo & 0,232 & 0,0090 & 0,246 & 0,0150 & $\mathrm{p}<0,01$ \\
\hline Al. pleura somito 3 & 0,0537 & 0,0035 & 0,0645 & 0,0047 & $\mathrm{p}<0,01$ \\
\hline Al. pleura somito 4 & 0,0625 & 0,0044 & 0,0751 & 0,0069 & $\mathrm{p}<0,01$ \\
\hline Al. pleura somito 5 & 0,0647 & 0,0035 & 0,0776 & 0,0072 & $\mathrm{p}<0,01$ \\
\hline L. pleura somito 2 & 0,1960 & 0,0087 & 0,2040 & 0,0087 & $\mathrm{p}<0,01$ \\
\hline
\end{tabular}


Tabla 4. Talla, forma sexual y tamaño de las oviposturas de las hembras ovígeras de $P$. pugnax. LEC = longitud estándar del cefalotórax.

Tabla 4. Lenght, sexual form and size of $P$. pugnax carrying female ovipositions. $\mathrm{LEC}=$ cephalothoraxic standard length.

\begin{tabular}{|c|c|c|c|c|}
\hline Muestreo & $\begin{array}{c}\text { Hembras } \\
\text { ovígeras }\end{array}$ & $\begin{array}{c}\text { LEC } \\
(\mathbf{m m})\end{array}$ & Intersexos & $\begin{array}{c}\text { Tamaño } \\
\text { ovipostura }\end{array}$ \\
\hline \multirow[t]{17}{*}{2} & 17 & 36,6 & 2 & 26 huevos \\
\hline & & 33,8 & 2 & 5 huevos \\
\hline & & 42,4 & 2 & 11 huevos \\
\hline & & 36,4 & 2 & 71 huevos \\
\hline & & 41,8 & 2 & 63 huevos \\
\hline & & 39,0 & 2 & 34 huevos \\
\hline & & 33,1 & 2 & 26 huevos \\
\hline & & 39,2 & 2 & 23 huevos \\
\hline & & 30,6 & 2 & 50 huevos \\
\hline & & 41,5 & 2 & 26 huevos \\
\hline & & 35,6 & 2 & 35 huevos \\
\hline & & 45,1 & 2 & 70 huevos \\
\hline & & 36,6 & 2 & 16 huevos \\
\hline & & 41,8 & 2 & 69 embriones \\
\hline & & 33,0 & 2 & 54 embriones \\
\hline & & 34,2 & 2 & 11 embriones \\
\hline & & 35,8 & 2 & 46 embriones \\
\hline \multirow[t]{2}{*}{3} & 2 & 40,8 & 2 & 8 embriones \\
\hline & & 35,5 & 2 & 19 juveniles 1 \\
\hline \multirow[t]{4}{*}{7} & 4 & 40,6 & 2 & 21 huevos \\
\hline & & 34,8 & 2 & 48 huevos \\
\hline & & 35,2 & 2 & 36 huevos \\
\hline & & 34,0 & 2 & 30 huevos \\
\hline
\end{tabular}

Distribución y proporción de sexos por galería

Durante los 7 muestreos realizados se revisaron 187 galerías. En 55 de ellas se encontraron dos ejemplares, que en la totalidad de los casos resultaron ser macho y hembra, de tallas superiores a $26 \mathrm{~mm}$ de longitud cefalotorácica. En otras 33 galerías se encontró un grupo de juveniles de tallas similares, acompañados de: una hembra (en 17 galerías), un macho (en una galería), un macho y una hembra (en 3 galerías), o sin compañía de ejemplares adultos (en 12 galerías). En las restantes 99 galerías se encontró un individuo en cada una de ellas. Al analizar la proporción de sexos, en 12 galerías que contenían un número comparativamente grande de juveniles (de tallas entre 6,6 y 18,4 mm de LEC), se observó en 8 de ellas una proporción de 1:1 y en las 4 restantes 1,8 machos por cada hembra.

\section{DISCUSION}

Todos los ejemplares de $P$. pugnax analizados en este trabajo presentaron gonoporos de macho y hembra. Este hecho confirma las observaciones que en tal sentido realizaron Faxon (1898), Lönnberg (1898), Hay (1905) y Turner (1935) en algunos especímenes de esta especie. Además, con respecto a este caracter $P$. pugnax se ajusta a las diagnosis de Parastacus, entregadas por Riek (1971) y por Hobbs (1974 y 1991). Sin embargo muchos de los especímenes estudiados (57,8\%), tienen gonoporos femeninos sellados por una membrana fuertemente calcificada (intersexo 1),

Tabla 5. Medias y desviaciones estándar del diámetro máximo de los ovocitos en hembras ovígeras y no ovígeras de $P$. pugnax. LEC = longitud estándard del cefalotórax.

Table 5. Means and standard deviations of the maximun diameter of $P$. pugnax ovocites in carrying and non carrying females. LEC $=$ cephalothoraxic standard length.

\begin{tabular}{|cccccccc|}
\hline Tipo de hembra & Hembras medidas & \multicolumn{3}{c}{ LEC $(\mathrm{mm})$} & \multicolumn{3}{c|}{ Diámetro máximo ovocitos $(\mathrm{mm})$} \\
& & $\overline{\mathbf{x}}$ & $\mathbf{\pm}$ D.S. & $\begin{array}{l}\text { ovocitos } \\
\text { medidos }\end{array}$ & $\overline{\mathbf{x}}$ & \pm D.S. \\
\hline Ovígera & 15 & 36,7 & 3,55 & 192 & 0,60 & 0,133 \\
No ovígera & 40 & 35,6 & 3,80 & 427 & 2,04 & 0,381 \\
\hline
\end{tabular}


mientras que el $42,2 \%$ restante poseen gonoporos femeninos cubiertos por una membrana no calcificada o sólo parcialmente calcificada (intersexo 2). Tal disposición había sido ya señalada por Faxon (1898), por Lönnberg (1898) y Hay (1905).

Las disecciones anatómicas y los cortes histológicos demostraron que estos ejemplares, pese a tener gonoporos supernumerarios, tienen una gónada única (masculina o femenina según el caso), de la cual emergen gonoductos de ambos sexos hacia los respectivos gonoporos. Si la gónada es un ovario, su histología femenina se mantiene en toda su extensión, sin rastros de tejido testicular, con oviductos bien desarrollados y espermiductos rudimentarios. Si por el contrario la gónada es un testículo, su estructura masculina es uniforme en toda la glándula, sin tejido ovárico, con vasos deferentes muy desarrollados y oviductos rudimentarios. Von Ihering (1893), Lönnberg (1898), Turner (1935) y Thompson (1982), disecaron algunos ejemplares adultos de $P$. brasiliensis, $P$. hassleri (= P. pugnax), $P$. saffordi, $P$. pilimanus y $P$. varicosus y en todos ellos observaron el mismo tipo de genitalia que se describe en este trabajo.

Si se consideran las evidencias aportadas por este trabajo y en particular los análisis morfométricos del abdómen, la distribución de frecuencia por clase de talla y las disecciones anatómicas, se puede señalar que bajo los $26 \mathrm{~mm}$ de LEC todos los individuos tienen una morfología externa indiferenciada que permite calificarlos como intersexos 1. Sin embargo sus gónadas ya están diferenciadas $(60,4 \%$ de machos y 39,6\% de hembras). Posteriormente se comprobó que esta diferenciación gonádica se manifiesta externamente en el alto de las papilas genitales masculinas. Los ejemplares con gónada masculina poseen una papila fálica más alta que aquella que presentan los especímenes con ovarios. Alrededor de los $26 \mathrm{~mm}$ de LEC (porque el 5,2\% de los intersexo 1 que se ubican sobre esta longitud cefalotorácica, resultaron ser hembras) las hembras gonádicas experimentarían la muda de pubertad, con lo cual sus gonoporos femeninos completarían su forma elipsoidal, abriéndose al exterior y quedando sólo cubiertos por una membrana fina que facilitaría la extrusión de sus ovocitos; adicionalmente sus abdomenes adquirirían una morfología reproductiva para permitir la incubación de sus huevos. Junto con esto, sus papilas fálicas adquirirían menor altura con respecto a las papilas fálicas de los machos (intersexo 2). Confirma lo anterior la presencia de hembras ovígeras sólo sobre los $30 \mathrm{~mm}$ de longitud cefalotorácica, todas ellas morfológicamente correspondientes a la categoría de intersexo 2 .

La presencia del morfo intersexo 2 (hembras), con tallas superiores a los $26 \mathrm{~mm}$ de LEC en todos los muestreos y con ovocitos en distintas etapas de vitelogénesis, permite afirmar que una vez que se logra la morfología reproductiva (o femenina), ella se mantendría por el resto de la vida. Lo cual también ocurre en hembras de Engaeus y P. nicoleti (Horwitz, 1988; Rudolph, 1995b).

Lönnberg (1898) señaló con ciertas reservas, derivadas del escaso número de ejemplares analizados, que en Parastacus pese a la presencia de gonoporos masculinos y femeninos en un mismo individuo, se podía diferenciar externamente el sexo por cuanto las hembras tenían un abdomen más ancho que los machos. Las evidencias aportadas por este trabajo indican que efectivamente es posible determinar externamente el sexo en $P$. pugnax. En ejemplares de tallas superiores a $26 \mathrm{~mm}$ lo anterior se puede hacer observando, al igual que en $P$. nicoleti, el grado de calcificación del gonoporo femenino, el ancho relativo del abdomen y el alto de las pleuras. Pero además, en esta especie el alto de las papilas fálicas es un caracter que también se puede utilizar para separar machos de hembras, especialmente en aquellos ejemplares que aún no experimentan la muda de pubertad (menores a 26 $\mathrm{mm}$ de LEC).

Entre los especímenes de talla inferior a los 25 $\mathrm{mm}$ de longitud cefalotorácica existe un número significativamente mayor de machos $(1,4: 1)$. Sobre esa talla se observa una ligera disminución del número de ellos $(1,2: 1)$, que por lo mismo no puede explicarse presumiendo un probable cambio de sexo. Sin embargo se aprecia una mayor proporción de hembras en las clases de tallas comprendidas entre 25 y 40 mm de LEC, lo cual podría resultar ventajoso para la reproducción de la especie puesto que muchas de estas hembras se encuentran en vitelogénesis secundaria y como consecuencia en condiciones de copular, como lo ratifica además el alto número de hembras en incubación registradas en estas clases de tallas.

La presencia de grupos de individuos al interior de 88 de las 187 galerías muestreadas confirma que esta especie vive en familia, al igual que otros Parastácidos excavadores (Riek, 1972; Horwitz y Richardson, 1986; Hobbs, 1988). Aún más, estos grupos se parecen bastante a los descritos por Rudolph (1995b) en $P$. nicoleti. La extracción de 
los ejemplares por succión, desde un sistema de galerías relativamente complejas, explicaría la ausencia en algunos grupos de uno o ambos progenitores, como así mismo las proporciones sexuales de 1,8:1 en favor de los machos observadas en algunos grupos de juveniles.

No se encontró ninguna evidencia de hermafroditismo en $P$. pugnax. Por ello llama la atención que por muchos años se le considerase una especie hermafrodita. Lönnberg (1898) no sólo se limitó a realizar un análisis anatómico de la genitalia de $P$. pugnax sino que además efectúo algunas observaciones microscópicas de macerados testiculares, en los cuales encontró ciertos cuerpos esféricos que por su forma y tamaño pensó que podrían corresponder a ovocitos inmaduros. Sobre esta base concluyó que esta especie podría ser hermafrodita parcial o rudimentaria. También generalizó al sostener que este tipo de hermafroditismo podría existir en todas las especies de Parastacus cuyos representantes contasen con gonoporos masculinos y femeninos. Hay (1905) sobre la base de las conclusiones de Lönnberg y sus propias observaciones de los orificios genitales de $P$. hassleri, $P$. defossus, $P$. saffordi y $P$. varicosus afirmó que el hermafroditismo descrito por Lönnberg debería ser la condición normal de estas especies. Lönnberg (1898) fue el primero y el único hasta 1935 que revisó, en forma minuciosa para la época, la genitalia de al menos una especie del género: P. hassleri (=P. pugnax). Por ello su afirmación de hermafroditismo en esta y probablemente en otras especies de Parastacus tuvo bastante influencia en autores posteriores, al punto que aúnque Turner (1935) y Thompson (1982) rechazaron la existencia de hermafroditismo, éste continuó siendo considerado como válido en ausencia de nuevas evidencias para explicar la presencia de gonoporos supernumerarios en este género (Hobbs, 1987). Es cierto que Rudolph (1995b) describió un hermafroditismo protándrico parcial en $P$. nicoleti. Sin embargo debe destacarse que ninguno de los autores citados anteriormente había revisado ejemplares de esta última especie. Observaciones microscópicas de macerados testiculares similares a los efectuados por Lönnberg (1898) muestran algunos cuerpos más o menos esféricos, aislados o en pequeños grupos. Sin embargo ellos corresponden a los acinos testiculares total o parcialmente separados por la maceración. Estos acinos probablemente hayan sido los cuerpos esféricos de estructura densa y parecidos a ovocitos que este autor creyó ver en testículos de $P$. pugnax.
Aparentemente la genitalia de $P$. pugnax desafía el control que la glándula androgénica ejerce sobre la diferenciación del sexo en Malacostraca. Charniaux-Cotton (1954) y muchos otros, han demostrado que la glándula androgénica de los Malacostráceos produce una hormona responsable de la diferenciación de los caracteres sexuales masculinos primarios y secundarios. En ausencia de hormona androgénica se produce autodiferenciación ovárica. Sin embargo, según Legrand y Juchault (1994) diferentes resultados relacionados con la gonadogénesis en Malacostraca muestran que la gónada, indiferenciada al momento del nacimiento, es bipotencial y presenta por lo general los primordios de ambos tipos de gonoductos. Por lo tanto cualquier factor, genético o epigenético, que altere el funcionamiento inicial de la glándula androgénica podría provocar el desarrollo de los primordios de oviductos presentes en los juveniles de ambos sexos. Lo anterior podría ser una buena explicación para la genitalia observada en los machos de $P$. pugnax, pero no explicaría aquella que existe en las hembras.

Ante la falta de evidencias de algún tipo de hermafroditismo en esta especie, resulta inevitable preguntarse acerca del valor adaptativo de una genitalia de este tipo. Difícil responder a esta interrogante con las escasas y fragmentarias evidencias que actualmente existen acerca de la sexualidad de los Parastacidae en general y de los excavadores en particular. Según Hobbs (1974 y 1988) los principales taxa actuales de Astacidea habrían evolucionado a partir de un ancestro gonocórico con desarrollo directo. Dicho ancestro ya habitaba en agua dulce (Scholtz, 1995). La familia Parastacidae sería monofilética (Riek, 1972; Patak y Baldwin, 1984) y habría evolucionado a lo largo de dos líneas independientes. Una constituída por excavadores moderados que habitan principalmente aguas permanentes y en los cuales los dáctilos de sus quelípodos se mueven en un plano horizontal (Euastacus, Euastacoides, Astacopsis, Cherax, Astacoides y Paranephrops) u oblicuo (Geocharax, Gramastacus, Parastacoides y Samastacus). La otra línea constituída por fuertes excavadores, en los cuales los dáctilos de sus quelípodos se mueven en un plano vertical (Parastacus, Engaeus, Engaewa y Tenuibranchiurus) (Riek, 1972). En Chile existen representantes de ambos grupos. Samastacus spinifrons (Philippi) es una especie gonocórica que habita ríos y lagos de la zona centro-sur de Chile y el lago Nahuel-huapi en la Argentina, que sólo 
manifiesta una escasa actividad excavadora (Rudolph, 1995a). P. pugnax y P. nicoleti en cambio habitan terrenos semipantanosos, en donde construyen galerías de hasta $2 \mathrm{~m}$ de profundidad (Kilian, 1959; Bahamonde y López, 1963) -no conectadas a cuerpos de agua permenentes- al interior de las cuales cumplen todo su ciclo vital, en grupos probablemente de tipo familiar. Sus poblaciones son relativamente pequeñas (Arias y Muñoz, 1991) y aisladas genéticamente debido a su escasa capacidad natatoria, a la ausencia de rutas y de larvas, y a la efectividad de las barreras (tipos de suelos) (Rudolph, 1995b). En estos aspectos la única diferencia conocida entre ambas especies es que $P$. pugnax tiene una distribución geográfica más amplia y al norte de aquella que presenta $P$. nicoleti. Por ello las poblaciones más septentrionales de $P$. pugnax habitan zonas con bajos índices de pluviosidad anual. Bajo estas condiciones $P$. nicolet $i$ habría evolucionado desde la condición gonocórica ancestral a un hermafroditismo protándrico parcial y $P$. pugnax se encontraría en una etapa temprana de evolución hacia algún tipo de hermafroditismo.

Las evidencias entregadas por este estudio y aquellas aportadas por Rudolph (1995b), señalan que mientras $P$. pugnax es una especie gonocórica, con individuos intersexo, $P$. nicoleti en cambio presenta dos tipos sexuales básicos, hembras primarias y hermafroditas protándricos. Por ello, aúnque las especies uruguayo-brasileñas de Parastacus presenten gonoporos supernumerarios no se les debe atribuir algunos de los patrones de sexualidad observados en las especies chilenas o algún otro, sin un estudio anatómico e histológico de sus respectivas gónadas y gonoductos.

\section{AGRADECIMIENTOS}

El autor agradece al Dr. Carlos Jara de la Universidad Austral de Chile, por la lectura crítica del manuscrito. A los Sres. Pedro Alvarado y Adalberto Gallegos, por su valiosa ayuda en terreno. A la Dirección de Investigación de la Universidad de Los Lagos, por el financiamiento otorgado a este trabajo (Proyecto 304.10-95).

\section{REFERENCIAS}

Amato, G. y G. Payen. 1976. Transplantations homoplastiques de testicules d'Ecrevisse, Pontastacus leptodactylus leptodactylus (Eschscholtz, 1823), en repos sexuel dans des mâles et des femelles normaux et épédonculés; résultats préliminaires. C. R. Acad. Sc. Paris, t. 283 Série D: 1783-1786.

Amato, G. y G. Payen. 1978. Mise en évidence du côntrole endocrine des différentes étapes de la spermatogenèse chez l'Ecrevisse Pontastacus leptodactylus leptodactylus (Eschscholtz, 1823), Crustacé, Décapode, Reptantia. Gen. Comp. Endocrinol., 36: 487-496.

Arias, P. y D. Muñoz. 1991. Antecedentes bioecológicos del camarón de vega Parastacus pugnax (Poeppig, 1835), en el área de Chillán. Agro-Ciencia, 7(2): 167-172.

Bahamonde, N. y M.T. López. 1963. Decápodos de aguas continentales en Chile. Invest. Zool. Chilenas, 10: 123-149.

Charniaux-Cotton, H. 1954. Découverte chez un Crustacé Amphipode (Orchestia gammarella) d' une glande endocrine responsable de la différenciation des caracteres sexuels primaires et secondaires mâles. Compt. R. Acad. Sci., 239: 780-782.

Faxon, W. 1898. Observations on the Astacidae in the United States Museum of Comparative Zoology, with descriptions of new species. Proc. U.S. Nat. Mus., 20: 643-694.

Hay, W.P. 1905. Instances of hermaphroditism in crayfishes. Smithson. Misc. Coll., 48: 222-228.

Hobbs, H.H. (Jr.). 1974. Synopsis of the families and genera of crayfishes (Crustacea:Decapoda). Smithson. Contrib. Zool, 164: 1-31.

Hobbs, H.H., Jr. 1987. A review of the crayfish genus Astacoides (Decapoda:Parastacidae). Smithson. Contrib. Zool., 443: 1-50.

Hobbs, H.H., Jr. 1988. Crayfish distribution, adaptive radiation and evolution. In: D.M. Holdich and R.S. Lowery (eds.) Freshwater crayfish: Biology, Management and Exploitation. Croom Helm, London, pp. 52-82.

Hobbs, H.H., Jr. 1989. An illustrated checklist of the American crayfishes (Decapoda: Astacidae, Cambaridae, and Parastacidae). Smithson. Contrib. Zool., 480: 236 pp. 
Hobbs, H.H., Jr. 1991. A new generic assignement for a South-American crayfish (Decapoda: Parastacidae) with revised diagnoses of the South American genera and comments on the parastacid mandible. Proc. Biol. Soc. Washington, 104(4): 800-811.

Holthuis, L.B. 1952. The Crustacea Decapoda Macrura of Chile. Reports of the Lund University Chile Expedition 1948-49, 5. Lunds Univ. Arssk. N.F. Avd. 2, Bd. 47, Nr. 10: 1-109.

Horwitz, P. 1988. Secondary sexual characteristics of females of the freshwater crayfish genus Engaeus (Decapoda: Parastacidae). Crustaceana, 54(1): 25-32.

Horwitz, P.H. y A.M. Richardson. 1986. An ecological classification of the burrows of australian freshwater crayfish. Aust. J. Mar. and Freshw. Res., 36: 237-242.

Kilian, E. 1959. La construcción de los tubos habitacionales del Parastacus nicoleti (Philippi, 1882). Fac. de Estudios Generales, Univ. Austral de Chile 1: 1-7.

Legrand, J-J. et. P. Juchault. 1994. Ontogenèse du sexe et physiologie sexuelle. In: J. Forest (ed.) Traité de Zoologie, Tome VII, Crustacés, Fascicule 1, Morphologie, physiologie, reproduction, embryologie. Masson, Paris, pp. 631-715.

Llönnberg, F. 1898. Some biological and anatomical facts concerning Parastacus. Zool. Anz., 21: 334-352.

Manning, R.B. y H.H. Hobbs, Jr. 1977. Decapoda. In: S.H. Hulbert (ed.) Biota acuática de Sudamérica austral. San Diego State University, San Diego, California, pp. 157-162.

Patak, A. y J. Baldwin. 1984. Electrophoretic and immunochemical comparisons of haemocyanins from australian freshwater crayfish (Family Parastacidae): Phylogenetic implications. J. Crustacean Biology, 4(4): 528-535.

Payen, G. 1973. Etude descriptive des principales étapes de la morphogenésis sexuelle chez un crustacé décapode a développement condensé, l'écrevisse Pontastacus leptodactylus leptodactylus (Eschscholtz, 1823). Ann. Embryol. Morph., 6(2): 179-206.

Philippi, R.A. 1882. Zoología chilena: Sobre los Astacus. An. Univ. Chile, 61: 624-628.
Riek, E. 1971. The freshwater crayfish of South America. Proc. Biol. Soc. Washington, 84: 129-136.

Riek, E. 1972. The phylogeny of the Parastacidae (Crustacea: Astacoidea), and description of a new genus of australian freshwater crayfishes. Aust. J. Zool., 20: 369-389.

Rudolph, E. 1990. Caracteres sexuales externos del camarón excavador Parastacus nicoleti (Philippi, 1882). Biota, 6: 19-34.

Rudolph, E.H. 1995a. A case of gynandromorphism in the freshwater crayfish Samastacus spinifrons (Philippi, 1882) (Decapoda: Parastacidae). Crustaceana, 68(6): 705-711.

Rudolph, E.H. 1995b. Partial protandric hermaphroditism in the burrowing crayfish Parastacus nicoleti (Philippi, 1882) (Decapoda: Parastacidae). J. Crustacean Biology, 15(4): 720-732.

Rudolph, E. y J. Ríos. 1987. Desarrollo ontogenético del camarón de las vegas Parastacus pugnax (Poeppig, 1835), en condiciones de laboratorio. Biota, 3: 45-58.

Rudolph, E., L. De la Fuente, M.E. Van Hasselt, A. Díaz y S. Dumenes. 1991. Composición química proximal de los tejidos comestibles y desperdicios de los camarones dulceacuícolas Samastacus spinifrons, Parastacus pugnax y Parastacus nicoleti (Crustacea: Decapoda: Parastacidae). Alimentos, 16(2): 23-29.

Scholtz, G. 1995. Ursprung und evolution der Flußkrebse (Crustacea, Astacida). Sber. Ges. Naturf. Freunden Berlin (N.F.) Bd., 34: 93-115.

Thompson, A. 1982. Contribución al estudio de la biología reproductiva de Parastacus varicosus Faxon, 1898 y de Parastacus pilimanus (Von Martens, 1869) (Crustacea: Decapoda: Parastacidae). Tesis de Licenciatura, Facultad de Humanidades y Ciencias, Univ. de la República. Montevideo, Uruguay.

Turner, C. 1935. The aberrant secondary sex characters of the crayfishes of the genus Cambarus. Amer. Midland Nat., 16: 863-882.

Von Ihering, H. 1893. Parastacus. Congrès Inter national de Zoologie. Moscou II: 43-49.

Von Martens, E. 1869. Südbrasilische Süss und Brack wasser Crustaceen nach den Sammlungen des Dr. Reinh Hensel. Archiv. Naturgesch., 35 (1): 1-37. 